\title{
Close binary EHB stars from SPY*ł†
}

\author{
R. Napiwotzki, C.A. Karl, T. Lisker and U. Heber \\ Dr. Remeis-Sternwarte, Universität Erlangen-Nürnberg, Bamberg, Germany \\ N. Christlieb and D. Reimers \\ Hamburger Sternwarte, Universität Hamburg, Hamburg, Germany \\ G. Nelemans \\ Institute of Astronomy, University of Cambridge, Cambridge, UK \\ D. Homeier \\ Dept. of Physics \& Astronomy, University of Georgia, Athens, USA
}

\begin{abstract}
We present the results of a radial velocity (RV) survey of 46 subdwarf B (sdB) and 23 helium-rich subdwarf O (He-sdO) stars. We detected $18(39 \%)$ new sdB binary systems, but only one $(4 \%)$ He-sdO binary. Orbital parameters of nine sdB and sdO binaries, derived from follow-up spectroscopy, are presented. Our results are compared with evolutionary scenarios and previous observational investigations.
\end{abstract}

Keywords: stars: horizontal branch, stars: evolution, stars: binaries

\section{Introduction}

In the standard picture (e.g. Heber, 1986) subdwarf B (sdB) stars are core helium burning stars on the extended horizontal branch (EHB). They consist of a He core with a mass of $\approx 0.48 M_{\odot}$, the canonical mass for a He core flash on the first red giant branch (RGB), and a thin, inert hydrogen shell. Evolutionary models of EHB stars calculated by Dorman et al. (1993) and D'Cruz et al. (1996) adopted an enhanced mass loss on the RGB, however, without specifying a particular mechanism.

Recent radial velocity (RV) surveys revealed that a large fraction of all sdB stars resides in close binaries (Saffer et al., 1998; Maxted et al., 2001; Edelmann et al., 2003). Han et al. (2002, 2003) performed a theoretical investigation of possible close binary channels for the formation of sdBs: stable Roche lobe overflow, common envelope ejection, and

\footnotetext{
* Based on data obtained at the Paranal Observatory of the European Southern Observatory for programs 165.H-0588, 167.D-0407, 70.D-0334(A), 71.D-0383(A)

$\dagger$ Based on observations collected at the German-Spanish Astronomical Center, Calar Alto, operated by the Max-Planck-Institut für Astronomie Heidelberg jointly with the Spanish National Commission for Astronomy

$\ddagger$ Based on data obtained with the WHT of the Isaac Newton Telescope operated on the island of La Palma by the Isaac Newton Group in the Spanish Observatory del Roque de los Muchachos of the Instituto de Astrofisica de Canarias
}

(c) 2018 Kluwer Academic Publishers. Printed in the Netherlands. 
merging of two He core white dwarf (WDs). They concluded that it is possible to explain all sdB stars as the result of close binary evolution.

The helium-rich subdwarf $\mathrm{O}$ stars (He-sdO) are hotter than sdBs, but have similar surface gravities. Their formation is still a mystery. In one scenario they are explained as an advanced stage of post-EHB evolution (Wesemael et al., 1982, Dreizler et al., 1990). Another scenario proposes the formation of He-sdOs via a phase as extreme He stars after the merging of two He core WDs (cf. discussion in Ahmad \& Jeffery, 2003).

SPY, the ESO Supernovae type Ia Progenitor surveY (Napiwotzki et al. 2001a, 2003), is a programme dedicated to search for short period binary WDs (double degenerates - DDs). The aim of SPY is the detection of DD progenitors of supernovae type Ia by means of a survey for RV variations. SN Ia progenitor candidates should be close enough to merge within one Hubble time due to gravitational wave radiation and the combined mass should exceed the Chandrasekhar limit for WDs. The SPY input catalogue collected WD candidates brighter than $B=16.5$ from a variety of source catalogues (cf. Napiwotzki et al., 2001a). The sdBs analyzed in this paper were selected from the Hamburg/ESO survey (Wisotzki et al., 1996), the McCook \& Sion (1999) WD catalogue, and the Hamburg Quasar Survey (Hagen et al., 1995).

As a by-product, SPY produced high accuracy RV measurements of more than $46 \mathrm{sdB}$ stars and $23 \mathrm{He}$-sdO stars, which were included in our sample because of misclassifications in the input catalogues. Thus SPY produces a large sample of known close binary sdB stars, offering an independent data set and allowing for a substantial improvement of the statistics. The He-sdO sample included in SPY is the first HesdO sample systematically checked for RV variations. This allows us to investigate the role of binarity for their formation and their possible link with the sdB stars.

\section{Results of the radial velocity survey}

SPY is carried out with the high-resolution spectrograph UVES at the UT2 telescope of ESO VLT. With our instrument setup we achieve nearly complete spectral coverage from $3200 \AA$ to $6650 \AA$ with only two $\approx 80 \AA$ wide gaps. Since SPY was implemented as a bad weather program, we used a wide $\left(2.1^{\prime \prime}\right)$ slit to minimize slit losses. The resulting spectral resolving power is $R=18500(0.36 \AA$ at $\mathrm{H} \alpha)$ or better, if seeing disks were smaller than the slit width. Due to the nature of the project, two spectra at different "random" epochs separated by at least one day are observed (cf. Napiwotzki et al., 2001a, for details). 


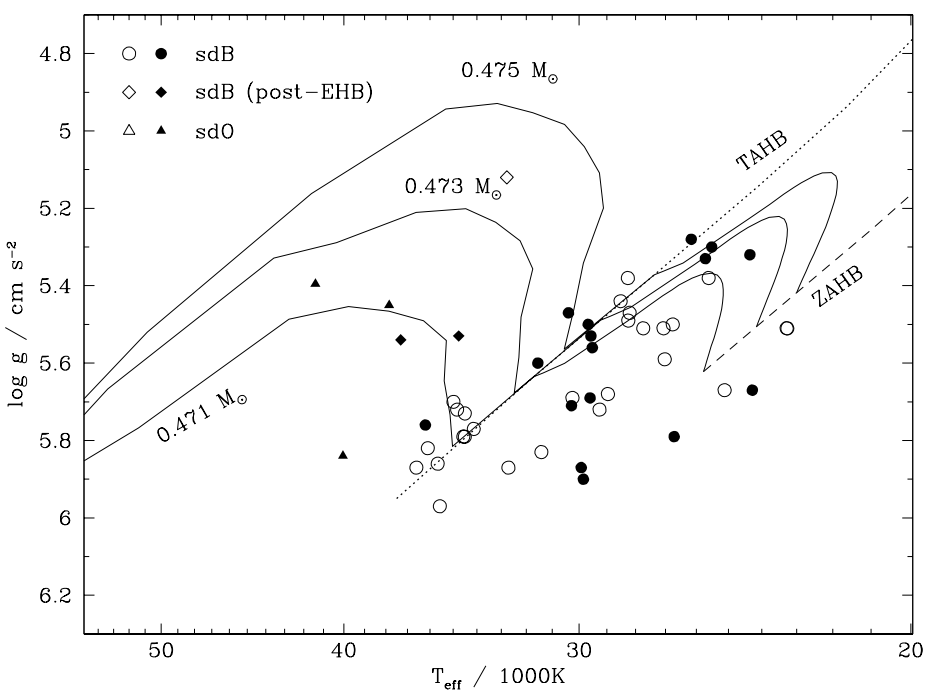

Figure 1. The sample of sdBs checked for RV variations in the temperature-gravity diagram (parameters from Lisker et al., 2003b). Filled symbols indicate RV variable binaries. The sdBs indicates as "post-EHB" are rejected by the "strip-selection" discussed in the text. Representative evolutionary tracks from Dorman et al. (1993) and the zero-age (ZAHB) and terminal-age (TAHB) horizontal branches are indicated.

The RV variations were measured differentially between both observed spectra with a cross correlation routine. This allowed to include all suitable lines $(\mathrm{H}, \mathrm{He}$, and metal lines) for the RV determination in a very flexible way. We routinely achieved a RV accuracy of $2 \mathrm{~km} / \mathrm{s}$ or better.

\subsection{The SDB SAMPle}

RV variations could be checked for 46 single-lined sdBs from the complete SPY sample (Lisker et al., 2003a, 2003b). Their distribution in the temperature-gravity plane is displayed in Fig. 1. Our RV measurements yielded 18 RV variable binaries, i.e. $39 \%$ of this sample. This indicates a high fraction of close binaries in the SPY sample, conforming the importance of binary channels for the formation of sdBs. However, somewhat surprisingly our result points to a lower fraction of detections than in the Maxted et al. (2001) sample, who found 58\% RV variable sdBs.

For a quantitative comparison with other samples and an evaluation of the true fraction of binaries one needs to know the detection efficiency, i.e. the chance that a binary escaped detection, because of unfavorable inclination angles or phasing of the observations. The de- 


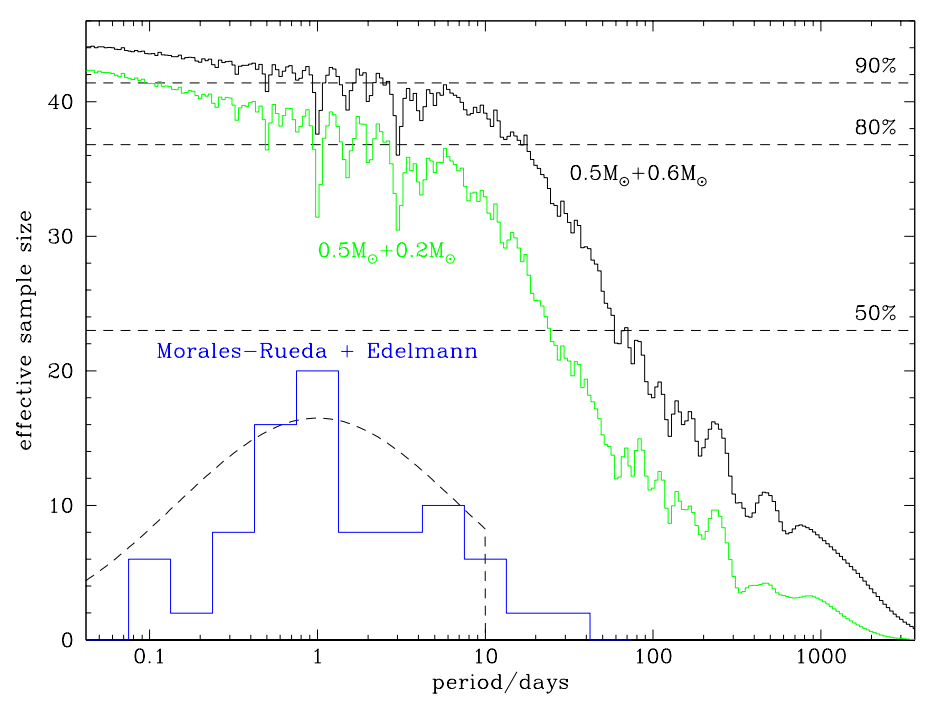

Figure 2. Detection efficiency as a function of orbital period for two different assumptions on the companion mass. For comparison we display the period distribution of sdBs for the combined sample of Morales-Rueda et al. (2003) and Edelmann et al. (2003). The dashed line indicates the period distribution adopted by Maxted et al. (2001) for estimating their detection efficiency.

tection efficiency obviously depends on the orbital period and the mass of the unseen companion. We performed a Monte Carlo simulation of our observed sample for two different choices of the mass of the invisible companion: $0.6 M_{\odot}$, the mass of a typical $\mathrm{WD}$, and $0.2 M_{\odot}$ for a typical low mass main sequence star. For every star we calculated detection probabilities taking into account the timing of exposures and the RV accuracy. Results for the sample were coadded yielding the effective sample size (i.e. the size of an equivalent sample with $100 \%$ binary detection rate) plotted in Fig. 2 as function of orbital period. For an alternative interpretation we indicate the relative percentage of the detection rate. The detection probabilities are quite high for short period systems, exceeding $90 \%$ for systems with a WD companion and periods shorter than one day.

However, to estimate the total numbers of binaries in the observed sample, one has to adopt a period distribution of the underlying binary population. We corrected the observed number of binaries for two different assumptions: 1) a truncated Gaussian centered on $P=$ 1d (see Fig. 2) and 2) a flat distribution covering the interval from $P=0.1 \mathrm{~d} \ldots 30 \mathrm{~d}$. The truncated Gaussian distribution corresponds to one of the distributions discussed by Maxted et al. (2001) and agrees 
Table I. Corrections for detection efficiency for the sdB and the He-sdO samples. Calculations were done for the two distributions discussed in the text, the canonical sdB mass of $0.5 M_{\odot}$, and different companion masses.

\begin{tabular}{llllll}
\hline Sample & $M_{\text {comp }} / M_{\odot}$ & distribution & $\begin{array}{l}\text { observed } \\
\text { rate }\end{array}$ & $\begin{array}{l}\text { efficiency } \\
\text { corrected } \\
\text { rate }\end{array}$ \\
\hline sdB & 0.2 & Gauss & $39 \%$ & $88 \%$ & $44 \%$ \\
& 0.2 & flat & $39 \%$ & $87 \%$ & $45 \%$ \\
\hline & 0.5 & Gauss & $39 \%$ & $93 \%$ & $42 \%$ \\
\hline & 0.6 & Gauss & $39 \%$ & $94 \%$ & $42 \%$ \\
& 0.6 & flat & $39 \%$ & $93 \%$ & $42 \%$ \\
\hline He-sdO & 0.2 & Gauss & $4 \%$ & $89 \%$ & $5 \%$ \\
& 0.2 & flat & $4 \%$ & $88 \%$ & $5 \%$ \\
\hline & 0.6 & Gauss & $4 \%$ & $94 \%$ & $5 \%$ \\
& 0.6 & flat & $4 \%$ & $94 \%$ & $5 \%$ \\
\hline
\end{tabular}

reasonably well with the current sample of sdBs with known orbital periods (Fig. 2). The flat distribution was chosen as some sort of worst case, because it gives high weight to the long period systems with relatively bad detection probabilities.

Results are detailed in Table I. The corrections are small and even in the worst case the likely binary fraction amounts to $45 \%$, i.e. 3 undetected systems are predicted. The entry for a companion mass of $0.5 M_{\odot}$ corresponds to corrections calculated by Maxted et al. (2001). Their corrections are small as well and they estimate a binary fraction of $69 \%$. If we compute the corrections for our sample in exactly the same way we derive $42 \%$. The difference between both samples is statistically significant.

However, selection criteria of both samples are slightly different. Maxted et al. excluded stars which lie significantly above the EHB from their sample ("strip-selection"). This criterion would exclude the three stars marked as "post-EHB" in Fig. 1 from our sample. Two of these three are binaries, which lowers the (corrected) binary frequency to $40 \%$. We excluded sdBs with spectral contamination from a main sequence companion, because these are usually not found in close binary systems, while Maxted et al. included two known sdBs with composite spectra. Moreover, our high resolution UVES spectra allow a more sensitive detection of cool companions which would have probably escaped detection in the lower resolution spectra of Maxted 
et al.. Including some or all composite systems into our sample would lower the frequency of close binaries in the SPY sample even further.

\subsection{The He-SdO sample}

A total of 23 single-lined He-sdOs was checked for RV variations We detected only one RV variable close binary in this sample, and this is a peculiar object: a double-lined system apparently consisting of two subdwarfs (Lisker et al., 2003a). The detection efficiency is very similar to the value of our sdB sample (see Table I). The corrected close binary fraction is $5 \%$, which is much lower than the percentage observed in any sdB sample, providing evidence that He-sdOs are not the progeny of sdBs.

\subsection{INDIVIDUAL BINARY SYSTEMS}

Detection of RV variations is only a start. Follow-up observations are necessary to derive important system parameters like orbital periods and amplitudes, which allow us to compute lower limits on the mass of the unseen companion via the mass function. This information will allow us to further constrain scenarios for the formation of sdBs.

We performed follow-up observations of detected sdB binaries at the $3.5 \mathrm{~m}$ telescope at Calar Alto, the William Herschel telescope at Roque de los Muchachos, and the ESO-VLT. Sufficient data for an unambiguous orbital solution is available for six sdB stars (including HE 1047-0436; Napiwotzki et al., 2001b). Details are given in Table II.

Table II contains data for three objects which are classified sdO. Unlike the He-sdOs discussed in Sect. 2.2 they are H-rich rather than He-rich with a spectral appearance not very different from the sdBs. A comparison with the parameter range covered by the SPY sdBs (Fig. 1) demonstrates that they represent nothing else than the hot end of the sdB distribution.

\section{Discussion and conclusions}

We have presented a RV survey of 46 sdBs and 23 He-sdOs for close binaries. We detected 18 (39\%) sdB and 1 (4\%) He-sdO binaries, respectively. Although we qualitatively confirm the results of Saffer et al. (1998) and Maxted et al. (2001), which indicate a high binary frequency among sdB stars, our quantitative results are at variance with Maxted et al. (2001), who detected 21 close binaries in a sample of $36 \mathrm{sdBs}$, i.e. $58 \%$. Differences in detection efficiency and selection effects cannot explain the discrepancy, as discussed in Sect. 2.1. After correction for 
Table II. Orbital parameters of sdB and sdO stars from SPY. We calculated the minimum mass $M_{2}(\mathrm{~min})$ from the mass function and the most probable mass for $i=52^{\circ}$. A primary mass of $0.5 M_{\odot}$ was adopted.

\begin{tabular}{lllll}
\hline Object & type & $P / \mathrm{d}$ & $M_{2}(\min ) / M_{\odot}$ & $M_{2}\left(i=52^{\circ}\right) / M_{\odot}$ \\
\hline WD 0048-202 & sdB & 7.45 & 0.33 & 0.47 \\
HE 0532-4503 & sdB & 0.2656 & 0.26 & 0.35 \\
HE 0929-0424 & sdB & 0.4400 & 0.38 & 0.54 \\
HE 1047-0436 & sdB & 1.2133 & 0.44 & 0.71 \\
HE 2135-3749 & sdB & 0.9241 & 0.35 & 0.50 \\
HE 2150-0238 & sdB & 1.322 & 0.50 & 0.73 \\
\hline HE 1059-2735 & sdO & 0.556 & 0.31 & 0.42 \\
HE 1115-0631 & sdO & 5.87 & 0.52 & 0.76 \\
HE 1318-2111 & sdO & 0.487 & 0.34 & 0.48 \\
\hline
\end{tabular}

undetected binaries with identical assumptions the estimated true rate of close binaries are $42 \%$ in the SPY sample vs. $69 \%$ in the Maxted et al. (2001) sample.

Stars from the SPY sample are typically much fainter than from the Maxted et al. sample, who concentrated on the brightest known sdB stars. Consequently $2 / 3$ of the sdBs from the SPY sample are more than $1 \mathrm{kpc}$ away from the Galactic plane, while only two of the Maxted et al. stars are. Thus we expect that a significant fraction of the SPY sdBs belong to the thick disk or the halo populations, which are both old and metal poor, while most of the Maxted et al. sdBs are probably members of the thin disk. Determination of population membership by a kinematical study of both samples would help to verify this explanation.

The very low number of close binaries in our He-sdO sample provides evidence that He-sdOs, as a class, are not successors of EHB stars. Formation of He-sdOs by merging of two He core WDs would be in agreement with our finding.

How do our sdB results compare with the binary population synthesis of Han et al. (2003)? Han et al. produced several simulated samples of sdBs resulting from their binary evolution channels with different parameter choices and presented a best fit sample (their simulation set 2). They could reproduce the properties of observed sdB binaries (from Morales-Rueda et al., 2003) and the distribution in the temperaturegravity plane (cf. Lisker et al., 2003a, 2003b). Observational selection effects against sdBs with visible cool companions (GK selection) and 
against stars above the EHB (strip selection) were simulated. They predict an observable binary frequency for their best fit model of $45 \%$ (the remainder resulting from merging), which compares well to our $40 \%$, if we apply the same criteria to the SPY sample.

However, two problems remain. Han et al. (2003) simulated a population with thin disk characteristics, which should be representative for the Maxted et al. (2001) sample, but this sample yielded a binary frequency of $69 \%$. On the other hand Han et al. (2003) predict a low binary frequency for stars above the EHB, because this region should contain many sdBs resulting from merging. Although a systematic observational investigation of stars in this region has still to be done, it appears that the binary frequency in this region is higher than expected (see Fig. 1) from the theoretical simulation. A further complication might arise if some or all of the mergers produce He-sdOs, instead. In summary the new theoretical simulations represent the properties of the observed sdB sample well, but some unsolved problems remain. Future investigations will help to decide, if these can be solved by minor adjustments or imply larger revisions.

\section{References}

Ahmad, A. \& Jeffery, C.S.: 2003, A\&A 402, 335

D'Cruz, N.L., Dorman, B., Rood, R.T., \& O'Connell, R.W.: 1996, ApJ 466, 359

Dorman, B., Rood, R.T., \& O'Connell, R.W.: 1993, ApJ 419, 596

Dreizler, S., Heber, U., Werner, K., Moehler, S., de Boer, K.S.: 1990, A\&\&A 235, 234

Edelmann, H., Heber, U., Lisker, T., Green, E.M.: 2003, Ap\&SSS, these proceedings

Hagen, H.-J., Groote, D., Engels, D., \& Reimers, D.: 1995, A\&AS 111, 195

Han, Z., Podsiadlowski, P., Maxted, P.F.L., Marsh, T.R., \& Ivanova, N.: 2002, MNRAS 336, 449

Han, Z., Podsiadlowski, P., Maxted, P.F.L., \& Marsh, T.R.: 2003, MNRAS 341, 669

Heber, U.: 1986, A\& A 155, 33

Lisker, T., Heber, H., Napiwotzki, R., et al.: 2003a, ApESSS, these proceedings

Lisker, T., Heber, H., Napiwotzki, R., et al.: 2003b, $A \mathscr{G} A$, submitted

Maxted, P.F.L., Heber, U., Marsh, T.R., \& North, R.C.: 2001, MNRAS 326, 1391

McCook, G.P. \& Sion, E.M.: 1999, ApJS 121, 1

Morales-Rueda, L., Maxted, P.F.L., Marsh, T.R., North, R.C., \& Heber, U.: 2003, MNRAS 338752

Napiwotzki, R., Christlieb, N., Drechsel, H., et al.: 2001a, $A N$ 322, 411

Napiwotzki, R., Edelmann, H., Heber, U., et al.: 2001b, A $\varepsilon A 378$, L17

Napiwotzki, R., Christlieb, N., Drechsel, H., et al.: 2003, ESO Messenger 112, 25

Saffer, R.A., Livio, M., \& Yungelson, L.R.: 1998, ApJ 502, 394

Wesemael, F., Winget, D.E., Cabot, W., van Horn, H.M., \& Fontaine, G.: 1982: ApJ 254,221

Wisotzki, L., Koehler, T., Groote, D., \& Reimers, D.: 1996, A\&AS 115, 227 